\title{
Discovery of Collocation Episodes in Spatiotemporal Data*
}

\author{
Huiping Cao, Nikos Mamoulis, and David W. Cheung \\ Department of Computer Science \\ The University of Hong Kong \\ Pokfulam Road, Hong Kong \\ \{hpcao,nikos, dcheung\}@cs.hku.hk
}

\begin{abstract}
Given a collection of trajectories of moving objects with different types (e.g., pumas, deers, vultures, etc.), we introduce the problem of discovering collocation episodes in them (e.g., if a puma is moving near a deer, then a vulture is also going to move close to the same deer with high probability within the next 3 minutes). Collocation episodes catch the inter-movement regularities among different types of objects. We formally define the problem of mining collocation episodes and propose two scaleable algorithms for its efficient solution. We empirically evaluate the performance of the proposed methods using synthetically generated data that emulate real-world object movements.
\end{abstract}

\section{Introduction}

The large volume of the spatiotemporal data, (i.e., moving objects trajectories) renders their manual analysis tedious and impossible. For their efficient analysis, spatiotemporal data mining [1] is proposed for the development and application of novel computational techniques. Given a trajectory database, our goal is to unveil inter-movement regularities among objects of different types, modeled as a sequence of collocation events. Consider an application that monitors the activities of animals (e.g., via sensors attached to them). An exemplary collocation episode for this application could be "Once we detect that a puma is moving close to a deer for 1 minute, we expect that a vulture will also move near to this deer in 3 minutes with high probability."

A collocation episode is in fact a sequence of spatiotemporal collocation events. Such events are sets of objects moving close to each other for some period. In addition, there is a particular object type (e.g., deer), called centric feature, which participates in a sequence of collocations (e.g., deer-puma, deer-vulture). Finally, in a valid instance of the episode the object that instantiates the common feature should be the same in all collocation instances (e.g., the same deer appears in both deer-puma and deer-vulture collocations). Our definition of a collocation event is a temporal extension of the spatial collocation defined in [6], which models the co-existence of a set of (non-spatial) features, such as environmental observations (humidity and pollution values), plant and animal types, etc., in a spatial neighborhood. E.g., pattern (wet, bamboo) means that, with high probability, we can find bamboo plants near places with high hu-

*Work supported by grant HKU 7142/04E from Hong Kong RGC. midity values. Existing methods that consider only spatial relationships between static features are not directly applicable for our problem, since (i) we require a temporal duration for a valid collocation event and (ii) we search for temporal episodes of such events. Our problem also has some similarity with episodes mining in sequence data [5], where frequent episodes are (partially or totally) ordered list of events. A sliding window $w$ is used to extract subsequences in the event series, and the contribution of every subsequence to each candidate episode's frequency is counted. However, the events in our episodes are complex collocations as opposed to simple categorical values. In addition, for a valid episode instance there must be a common feature instantiation ${ }^{1}$ (centric feature requirement), as opposed to an appearance of any event of the same type in [5].

In view of the challenges in this problem, we propose a two-step framework for mining collocation episodes. First, we apply a hash-based technique to efficiently retrieve the object pairs, whose trajectories are close during some periods and identify these intervals. Then, we provide two collocation episode mining algorithms (one Apriori-based approach and one that is based on the vertical mining paradigm) and some pruning techniques to improve the mining efficiency. Finally, we empirically evaluate the performance of the proposed methods using synthetically generated data. In the remainder of the paper, we introduce some related work, formally define the problem, outline our algorithms, and present an experimental evaluation for them.

\section{Related Work}

Besides the work reviewed in Section 1, our work is also related to pattern discovery in one-dimensional time series, e.g., [4] etc. Nevertheless, these problems differ in three main aspects from our work; (i) the pattern/rule element is just a symbol or an event, while our pattern unit is a topological structure with a temporal duration; (ii) patterns are defined based on a single time series, but our patterns are based on relationships among different sequences; and (iii) temporal and time-series data mining is usually based on predefined categorization of $1 \mathrm{D}$ values, whereas we work on a continuous (spatiotemporal) data space. In addition, several efforts have been made to extend spatial collocation patterns [6] to contain temporal aspects towards different directions. E.g., from spatiotemporal data, [9] searches for evolving collocations, which in nature are pattern components in our context, while [8] discovers topological patterns (without tempo-

\footnotetext{
${ }^{1}$ Note that two trajectories of the same type (e.g., deer) may correspond to different objects (e.g., two different deers).
} 
ral order). Finally, a related piece of work to our problem is [3], where spatiotemporal pattern queries are proposed and studied. An intuitive example of such a query is "find the moving object that is close to location $A$ at time $t_{1}$, and then moves to region $C$ during time interval $\left[t_{3}, t_{4}\right]$ ". The main differences of our work are (i) we automatically identify frequent patterns that relate the movement of objects, instead of posing explicit queries and (ii) our patterns relate two or more trajectories (that are feature instances), instead of searching for trajectories that follow a specific "route" specified by a temporal sequence of static regions.

\section{Problem Definition}

This section formally defines the spatiotemporal collocation episodes by gracefully combining the concept of episode in event sequences and collocation in spatial databases.

\subsection{Spatiotemporal sequences and close subsequences}

A spatiotemporal sequence $\mathcal{S}$ is the trajectory of a moving object. Formally, it is an ordered list of location-time pairs $\left(l_{0}, t_{0}\right),\left(l_{1}, t_{1}\right), \cdots,\left(l_{m-1}, t_{m-1}\right)$ where $t_{i}<t_{j}$ if $i<j$ $(i, j \in[0, m))$. The pair $\left(l_{i}, t_{i}\right)$ denotes that the object was at location $l_{i}$ at time $t_{i}$. In practice, $l_{i}$ is a 2D position $\left(x_{i}, y_{i}\right)$, and $t_{i}$ records the time represented in time units (e.g., one minute is a time unit). In the following discussion, the subscript of a location implicitly refers to its timestamp (i.e., $l_{i}$ implies the location at time $t_{i}$ ).

Given $n(n \geq 1)$ objects $o_{1}, o_{2}, \cdots, o_{n}$, the trajectory of object $o_{i}$ is denoted by $\mathcal{S}_{i}$. Figure 1 plots three exemplary sequences $\mathcal{S}_{P}, \mathcal{S}_{D}$ and $\mathcal{S}_{V}$ (abbreviating $\mathcal{S}_{P u m a}, \mathcal{S}_{\text {Deer }}$, and $\mathcal{S}_{\text {Vulture }}$, respectively). For illustration purposes, we use 1D values to represent spatial locations, however, our discussion extends naturally to the multidimensional space.

A subsequence $s$ of $\mathcal{S}$ is a list of continuous locationtime pairs of $\mathcal{S}:\left(l_{i_{1}}, t_{i_{1}}\right),\left(l_{i_{2}}, t_{i_{2}}\right), \cdots,\left(l_{i_{q}}, t_{i_{q}}\right)$, where for $\forall j \in[1, q], l_{i_{j}}$ is in $\mathcal{S}$ and $t_{i_{j}}+1=t_{i_{j+1}}$. The starting (ending) time of $s$ is denoted by $s . t_{s}\left(s . t_{e}\right)$. For a complete sequence $\mathcal{S}$ with $m$ positions, $\mathcal{S}$. $t_{s}=t_{0}$ and $\mathcal{S} . t_{e}=t_{m-1}+1$.

Definition $1 A$ window is a time interval $\left[t_{s}, t_{e}\right)$. The time span (or length) of a window $\left[t_{s}, t_{e}\right.$ ) is $t_{e}-t_{s}$. A window with time span $w$ is called a $w$-window.

Definition 2 A subsequence $s$ is on window $\left[t_{s}, t_{e}\right)$ if s.t $t_{s}=$ $t_{s}$ and $s . t_{e}=t_{e}$. A subsequence is called a $w$-subsequence if it is on a w-window. Two subsequences $s_{i} \in \mathcal{S}_{i}$ and $s_{j} \in \mathcal{S}_{j}$ are concurrent subsequences if they are on the same window.

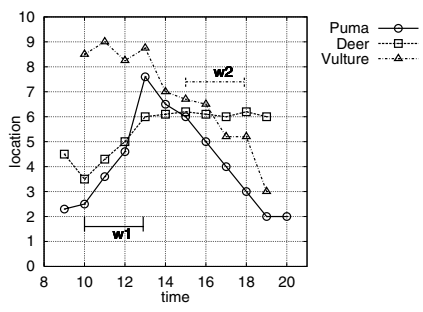

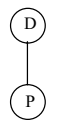

(a)

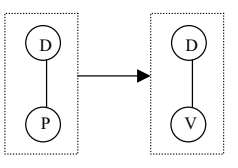

(b)
Figure 1: Example of trajectories $\begin{aligned} & \text { Figure 2: Collocat } \\ & \text { unit and episode }\end{aligned}$ and windows

Example: Figure 1 shows two windows with time span 3: $\mathbf{w} \mathbf{1}=[10,13)$ and $\mathbf{w} 2=[15,18)$. For $\mathcal{S}_{D}$, the two subsequences on $\mathbf{w} 1$ and $\mathbf{w} 2$ are $s_{D_{1}}=(3.5,10),(4.3,11),(5.0,12)$ and $s_{D_{2}}=(6.2,15),(6.1,16),(6.0,17)$. For $\mathcal{S}_{P}$, the subsequence on w1 is $s_{P_{1}}=(2.5,10),(3.6,11),(4.6,12)$, while on w2 we have $s_{V_{2}}=(6.7,15),(6.5,16),(5.2,17)$ from $\mathcal{S}_{V} \cdot s_{D_{1}}$ and $s_{P_{1}}$ (also: $s_{D_{2}}$ and $s_{V_{2}}$ ) are concurrent subsequences. $\mathcal{S}_{D}$ is on window $[9,20)$ and has eight $\left(t_{e}-t_{s}-w=\right.$ $20-9-3) 3$-subsequences, the second of which is on w1.

We define the closeness of two concurrent subsequences, using an aggregate function aggDist for their element-toelement distances. Typically, aggDist can be the maximum (max) or average (avg) of the component distances. Assuming that $s_{i}$ and $s_{j}$ are both on window $\left[t_{s}, t_{e}\right)$, and dist is some atomic distance function (e.g., Euclidean distance), $\max \operatorname{Dist}\left(s_{i}, s_{j}\right)=\max _{t_{s} \leq i_{t}=j_{t}<t_{e}}\left\{\operatorname{dist}\left(l_{i_{t}}, l_{j_{t}}\right)\right\}$ and $\operatorname{avgDist}\left(s_{i}, s_{j}\right)=\frac{\sum_{t_{s} \leq i_{t}=j_{t}<t_{e}} \operatorname{dist}\left(l_{i_{t}}, l_{j_{t}}\right)}{t_{e}-t_{s}}$. A distance threshold $\epsilon$ is used to model closeness:

Definition 3 Two concurrent subsequences $s_{i}$ and $s_{j}$ are close, denoted by close $\left(s_{i}, s_{j}\right)$, if aggDist $\left(s_{i}, s_{j}\right) \leq \epsilon$.

Example: Assuming $\epsilon=2.5$ and $a g g$ Dist $=\max$ Dist, the two concurrent subsequences $s_{D_{1}}$ and $s_{P_{1}}$ on window w1 of Figure 1 are close to each other since for $\forall i_{t}=j_{t} \in[10,13)$, the location pair $l_{i_{t}} \in s_{i}$ and $l_{j_{t}} \in s_{j}$ satisfies $\operatorname{dist}\left(l_{i_{t}}, l_{j_{t}}\right) \leq \epsilon$.

\subsection{Spatiotemporal collocations and episodes thereof}

Let $\mathcal{F}$ be a set of moving object types (e.g., different animals). Given a database of object trajectories, the type (or feature) of an object $o_{i}$ is denoted by type $\left(o_{i}\right)$, such that type $\left(o_{i}\right) \in \mathcal{F}$. In general, the number of objects $n$ in the database can be larger than the number $|\mathcal{F}|$ of types in $\mathcal{F}$; i.e., more than one object may belong to the same object type.

Definition 4 A spatiotemporal collocation unit $g$ (simply unit) is an undirected graph $(V, E)$ where each vertex in $g . V$ is an object type in $\mathcal{F}$. The length of the unit $g$ is the number of vertices $|g . V|$ in it. Given a unit time span $w$, a valid instance $I_{g}$ of unit $g=(V, E)$, where $V=\left\{f_{1}, f_{2}, \ldots, f_{|V|}\right\}$, is a set of concurrent $w$-subsequences $\left\{s_{1}, s_{2}, \ldots, s_{|V|}\right\}$ on a window $\left[t_{s}, t_{e}\right)$ such that (i) $s_{i}$ is of type $f_{i},(1 \leq i \leq|V|)$ and (ii) if $\left(f_{i}, f_{j}\right) \in E$ then close $\left(s_{i}, s_{j}\right)$.

The starting (ending) time of $I_{g}$ is denoted by $I_{g} \cdot t_{s}\left(I_{g} \cdot t_{e}\right)$. For example, the two concurrent window trajectories $s_{D_{1}}$ and $s_{P_{1}}$ in Figure 1 is an instance of the collocation unit in Figure $2 \mathrm{a}$, and the related window is $[10,13)$ (i.e., w1).

Definition $5 A$ spatiotemporal collocation pattern (or episode) $P$ is an ordered list of spatiotemporal collocation units: $g_{1} g_{2} \cdots g_{\ell}$ where $\cap_{i=1}^{\ell}\left(g_{i} . V\right) \neq \varnothing$.

The object types in $\cap_{i=1}^{\ell}\left(g_{i} . V\right)$, are called the reference types (features) of pattern $P$. The length of the pattern $P$ is defined by $\sum_{i=1}^{\ell}\left|g_{i} . V\right|$. A pattern with length $k$ is called a $k$-pattern. In this paper, we only consider the case that $\left|\cap_{i=1}^{\ell}\left(g_{i} . V\right)\right|=1$, and we denote the common (reference) object type as $f_{r}$. The reference object type $f_{r}$ is also called the centric feature of the pattern. Thus, we can also represent a pattern in the form $\left(f_{r}, g_{1} . V-f_{r}\right) \rightarrow \cdots \rightarrow\left(f_{r}, g_{\ell} . V-f_{r}\right)$, where the underlined feature is the reference feature.

Example: Figure 2b shows a 4-collocation episode, indicating that when a deer and a puma are close during $w=3$ time units, a vulture will come close to this deer later. This episode's common feature is $D$ and can also be represented by $(\underline{D}, P) \rightarrow(\underline{D}, V)$.

Definition 6 Given a maximum pattern time span $W$, a valid instance $I_{P}$ for a pattern $P=g_{1} g_{2} \cdots g_{\ell}$, is a sequence of valid unit instances $I_{g_{1}} I_{g_{2}} \cdots I_{g_{\ell}}$ such that (i) in all unit 
instances the reference feature $f_{r}$ is instantiated by a subsequence of the same object sequence, (ii) for every $i<j$, $I_{g_{i}} \cdot t_{e} \leq I_{g_{j}} \cdot t_{s}$, and (iii) $I_{g_{\ell}} \cdot t_{e}-I_{g_{1}} \cdot t_{s} \leq W$.

Example: Let $\epsilon=2.5, w=3$, and $W=8$. In Figure 1, we can identify a valid instance of the episode of Figure $2 \mathrm{~b}$. In specific, $s_{D_{1}}$ and $s_{P_{1}}\left(s_{D_{2}}\right.$ and $\left.s_{V_{2}}\right)$ instantiate the first (second) unit of the pattern. In addition, $s_{D_{1}}$ and $s_{D_{2}}$ instantiate the common feature $D$ in both units and they are parts of the same trajectory. Furthermore, $I_{g_{1}} . t_{e}<I_{g_{2}} . t_{s}$, since the end point of $\mathbf{w} 1$ is before $\mathbf{w} 2$. Finally, $I_{g_{2}} \cdot t_{e}-I_{g_{1}} \cdot t_{s}=18-10 \leq W$.

Given the maximal episode time span $W$, we say that a $W$-window $\left[t_{s}, t_{e}\right)$ covers a pattern instance, if the time span of the instance $\left[I_{g_{1}} \cdot t_{s}, I_{g_{\ell}} \cdot t_{e}\right)$ satisfies $t_{s} \leq I_{g_{1}} \cdot t_{s}$ and $I_{g_{\ell}} \cdot t_{e} \leq t_{e}$. We use $\left|I_{P}\right|$ to denote the number of $W$ windows, which cover at least one instance of pattern $P$.

Definition 7 Pattern $P=g_{1} g_{2} \cdots g_{p}$ is a superpattern of $P^{\prime}=g_{1}^{\prime} g_{2}^{\prime} \cdots g_{q}^{\prime}$ if (i) P. $f_{r}=P^{\prime} . f_{r}$ and (ii) there exists $q$ units $g_{i_{1}} g_{i_{2}} \cdots g_{i_{q}}\left(1 \leq i_{j}<i_{j+1} \leq p, 1 \leq j<q\right)$ of $P$ such that $g_{j}^{\prime} . V \subseteq g_{i_{j}} . V$ and $g_{j}^{\prime} . E \subseteq g_{i_{j}} . E$ for $\forall j \in[1, q] . P^{\prime}$ is a subpattern of $P$.

For Example, $P=(\underline{A}, B, C) \rightarrow(\underline{A}, C, D) \rightarrow(\underline{A}, E)$ is a superpattern of $P^{\prime}=(\underline{A}, B) \rightarrow(\underline{A}, \underline{C}, D)$

To measure the interestingness of a collocation episode, we use the reference type as the key factor since it does not make sense to overcount the same instance of the reference feature (e.g., deer) with different instances of the other object types (e.g., puma, vulture) in the pattern. In addition, we consider all possible time windows $W$, where the pattern may appear.

Definition 8 The frequency of a pattern $P$ with reference object type $f_{r}$ is $f r(P, w, W)=\frac{\left|I_{P}\right|}{\sum_{\text {type }\left(o_{i}\right)=f_{r}} \mid \text { win }}$.

Here, win is the total number of $W$-subsequences in all $\mathcal{S}_{i}$-s where type $\left(o_{i}\right)=f_{r}$.

Let min_sup be the minimum frequent threshold that the users are interested, one pattern $P$ is frequent if $f r(P, w, W) \geq$ min_sup.

Problem Definition: Given a database of trajectories $\mathcal{S}_{1}, \cdots, \mathcal{S}_{n}$ of $n$ moving objects, each with type $\left(o_{i}\right) \in \mathcal{F}$, discover all the frequent spatiotemporal collocations, subject to $\epsilon$, a closeness duration window length $w$, a maximum pattern window length $W$, and a frequency threshold min_sup $\in[0,1)$.

\section{Algorithms}

To find the collocation episodes, the main tasks are: (i) identify the types of objects that move closely to each other, and (ii) find on which $W$-windows this closeness is observed.

\subsection{Finding close subsequences}

The first mining phase aims at discovering object pairs of different types $\left(f_{i}, f_{j}\right)$ that have close concurrent subsequences. The ultimate objective is to identify the collocation units that may form longer episodes. For this, we scan each $\mathcal{S}_{i}$ of type $f_{i}$ to identify its $w$-subsequences that are close to object subsequences of different type $f_{j}, j \neq i$. We store the starting position $t_{s}$ of each such window $\left[t_{s}, t_{s}+w\right)$ along with the set of object types close to $\mathcal{S}_{i}$, during $\left[t_{s}, t_{s}+w\right)$. Eventually, each trajectory $\mathcal{S}_{i}$ is converted to a feature sequence of the form $\mathcal{S}_{i}^{f}=\left\{\left\langle F_{1}, t_{1}\right\rangle,\left\langle F_{2}, t_{2}\right\rangle, \ldots,\left\langle F_{m}, t_{m}\right\rangle\right\}$,

where $F_{s}$ is the set of object types other than $f_{i}$ close to the $w$-subsequence of $\mathcal{S}_{i}$ that starts at time $t_{s}$.

A naive method for the computation of $\mathcal{S}_{i}^{f}$ for each $\mathcal{S}_{i}$, is to scan all the other sequences in order to identify the windows and feature-sets in each $\mathcal{S}_{i}^{f}$. We now present a hashbased technique that achieves this goal in two database scans only and is shown in Figure 3. In the first pass, all data are hashed to a 3D grid in the trajectory space (Line 1), where $\mathcal{G}$ and $\mathcal{T}$ are the projected length of each cell on spatial and temporal dimension, and are chosen to be $\epsilon$ and $w$ respectively. Then, the algorithm performs a pass over the hashed data by examining only one hyperplane of cells at a time, corresponding to a $w$-period. For each cell $g c$, the neighboring cells in the spatial dimensions having the same temporal coordinates as $g c$ are examined. In the 2D example of Figure 4 , for cell $g c$, starting at time $t_{s}$, and for the trajectory $\mathcal{S}_{i}$ (partially) inside $g c$, the (shaded) cells are checked for possible containment of subsequences which are partially close to $\mathcal{S}_{i}$. Note that $\mathcal{S}_{j}$ is close to $\mathcal{S}_{i}$ at time $t_{s}+2$, which means that this closeness relationship can be extended to a subsequence closeness in cells of time span $\left[t_{s}+w, t_{s}+2 w\right)$. Thus, the algorithm for each $\mathcal{S}_{i}$ buffers such partially close subsequences that can be extended to $\mathcal{S}_{i}^{f}$ elements. When the next hyperplane of cells is examined, the partial closeness results are examined for potential extension and inclusion to $\mathcal{S}_{i}^{f}$, along with generation of new partial results. The sorting of cell contents by time facilitates the fast identification and extension of partial closeness results, in a merge-join fashion.

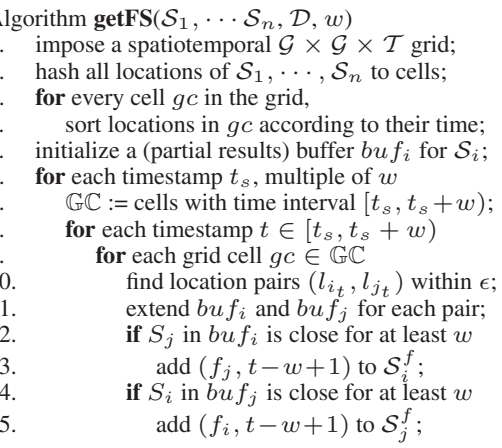

Figure 3: Hash-based computation of close feature sets

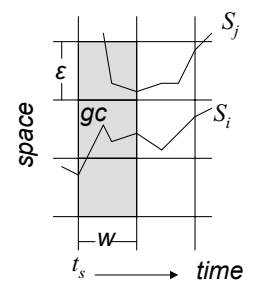

Figure 4: Example of hashing

\subsection{Discovery of collocation episodes}

In this section, we show two algorithms to discover frequent collocations, based on different usage of the transformed sequence of feature sets.

\subsubsection{Pattern extraction from sequences of feature sets}

The first algorithm Apriori shown in Figure 5 finds the collocations level-by-level. It takes as input the close feature sets $\mathcal{S}_{i}^{f}$ found for each $\mathcal{S}_{i}$, the minimum frequency mincnt $f_{f_{r}}$ $(=$ min_sup $\times \mid$ win $\mid)$ for an episode to be frequent. First, the $\mathcal{S}_{i}^{f}$ 's are partitioned to $|\mathcal{F}|$ groups, one for each different $f_{i} \in \mathcal{F}$. Thus, the group $\mathbb{S}_{f_{r}}$, for feature $f_{r}$ is used to find the patterns, having $f_{r}$ as their reference feature. We note that the apriori property holds for frequent episodes, i.e., if $P^{\prime}$ is a superpattern of $P$, then $f r(P, w, W) \leq f r\left(P^{\prime}, w, W\right)$. In this algorithm, when we measure the length of a pattern, we exclude the reference feature $f_{r}$ from the units, since it is implicit. For example, a 3 -candidate $\left(f_{i}\right) \rightarrow\left(f_{j}, f_{k}\right)$ represents a real 5-candidate $\left(\underline{f_{r}}, f_{i}\right) \rightarrow\left(\underline{f_{r}}, f_{j}, f_{k}\right)$. 
Function gen_cand, used to generate the $\ell$-candidates from $(\ell-1)$-patterns, is exactly as that in sequential pattern mining [7], so we will not discuss it in detail. The

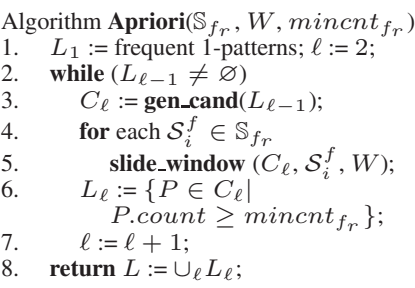

Figure 5: Apriori-based algorithm

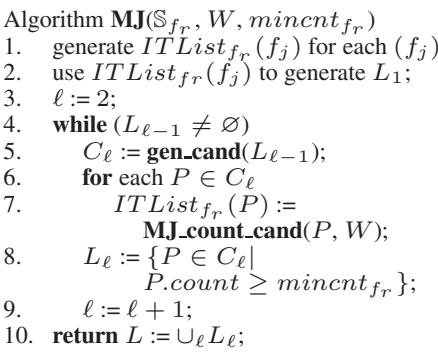

10. return $L:=\cup_{\ell} L_{\ell}$

Figure 6: Merge join algorithm

patterns excluding the reference features are similar to the sequential patterns in transactional databases [7]. However, counting the support of our patterns is different, since we consider all positions of a sliding window, whereas for sequential patterns each transaction sequence contributes one or none to a sequential pattern (depending on whether the sequence is a superpattern of it or not). Function slide_window is used to count $\left|I_{c}\right|$ (the number of windows that contain valid instances of $c$ ) for each candidate $c \in C_{\ell}$ from a transformed sequence $\mathcal{S}_{i}^{f} \in \mathbb{S}_{f_{r}}$. In brief, the idea is to slide a $W$-window over $\mathcal{S}_{i}^{f}$ to get a subsequence of feature sets. For each subsequence $s$ on a $W$-window, we find the candidates that have a valid instance, which is covered (i.e., supported) by $s$, and increase their count. Sliding window counting for event episodes has also been proposed in [5], however, the valid instances in our case are more difficult to count, because of the constraint that one collocation unit instance should end before the beginning of the next one (see condition (ii) in Definition 6). For example, assuming $w=3$ and $\left.\mathcal{S}_{i}^{f}=\left\{\left\langle\left(f_{1}, f_{2}\right), 10\right\rangle,\left\langle\left(f_{1}, f_{3}\right), 11\right\rangle,\left\langle\left(f_{4}\right), 14\right)\right\rangle\right\}$, pattern $c_{1}=\left(f_{1}, f_{2}\right) \rightarrow\left(f_{4}\right)$ is supported by $\mathcal{S}_{i}^{f}$, but pattern $c_{2}=\left(f_{1}, f_{2}\right) \rightarrow\left(f_{3}\right)$ is not, since $f_{3}$ is close to the reference feature at time 11 , which is before the end of $f_{2}(10+w=13)$. In simple words, in a valid pattern instance, the collocation unit instances should not overlap in time.

Optimizing the support counting While sliding a $W$ window over the transformed sequence, if the subsequence of $\mathcal{S}_{i}^{f}$ covered by the window remains the same compared to the previous window position, the set of candidates supported by the window does not change. As a result, we examine only positions of the $W$ window, where either (i) a feature-set $F$ is included in the window for the first time or (ii) $F$ ceases to be included in the window (compared to the previous position). E.g., let $w=3, W=8$, and $\mathcal{S}_{i}^{f}=$ $\left.\left\{\left\langle\left(f_{1}, f_{2}\right), 10\right\rangle,\left\langle\left(f_{1}, f_{3}\right), 11\right\rangle,\left\langle\left(f_{4}\right), 14\right)\right\rangle\right\}$. Since only three windows, $[5,13),[6,14),[9,17)$, correspond to the event of a feature-set entering the sliding window, and two windows, $[11,19),[12,20)$, correspond to the event that a feature-set leaves the window, we just need to examine these five windows. Each feature-set $\left\langle F_{i}, t_{i}\right\rangle \in \mathcal{S}_{i}^{f}$ affects two positions of window $\left[t_{s}, t_{e}\right.$ ); the one with $t_{e}=t_{i}+w$ (where $F_{i}$ enters the window) and the one with $t_{s}=t_{i}+1$ (where $F_{i}$ leaves it). As a result, the cost of examining a feature-set sequence $\mathcal{S}_{i}^{f}$ becomes proportional to $\left|\mathcal{S}_{i}^{f}\right|$, instead of the number of window positions (which normally is much larger).

Figure 7 shows in detail this optimized counting method applied for each $\mathcal{S}_{i}^{f}$. To avoid overcounting a pattern having more than one instance at a window position, when we detect a valid instance, we add to its support only for the window positions, where previous instances are not valid. For this, we maintain a variable c.last for each candidate (initialized to -1 ), indicating the last known position of $W$, having an instance of $c$. In addition, the algorithm keeps track of the feature-sets $f s$ contained in $W$. Whenever a feature-set $F$ exits the sliding window, it is removed from $f s$. If a new $F$ enters $f s$, we search for candidates for which the last unit is instantiated by some features in $F$ (instances not affected by $F$ are identified at earlier positions of $W$ ). I.e., only candidates, for which the features in the last unit are all contained in $F$, are checked for instantiation. For each candidate, if we detect a valid instance at the current window position, we look for the pattern instance with the latest starting time $I_{g_{1}} \cdot t_{s}$. The support of the candidate is then updated with the number of window positions $I_{g_{1}} \cdot t_{s}-t_{s}+1$, during which the pattern instance remains valid (when $t_{s}>I_{g_{1}} . t_{s}$, the instance becomes outdated). Finally, if some window positions were already counted due to the last detected pattern for $c$, i.e., if c.last $\geq t_{s}$, then we add $I_{g_{1}} . t_{s}-$ cllast to $c$.count (instead of $\left.I_{g_{1}} \cdot t_{s}-t_{s}+1\right)$, in order not to overcount the specific candidate.

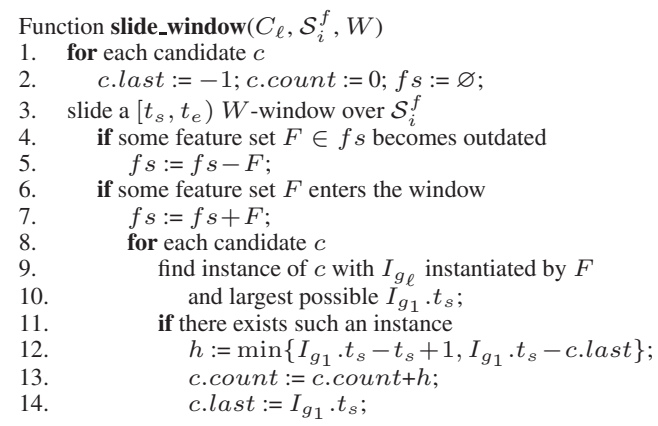

Figure 7: Optimized support counting

\subsubsection{Pattern extraction by joining instances of patterns}

Our second algorithm follows the vertical mining paradigm. Instead of scanning the $\mathcal{S}_{i}^{f}$ lists multiple times, while generating and counting candidates level-by-level, we keep track of the details about the instances of the patterns and join them to produce the instances of their superpatterns.

Figure 6 shows a pseudocode for this merge join $(\mathbf{M J})$ algorithm. First (Line 1), we scan the $\mathcal{S}_{i}^{f}$ lists, to produce the instance lists (ITLists) of all 1-patterns. For each reference feature $f_{r}$, all $\mathcal{S}_{i}^{f} \in \mathbb{S}_{f_{r}}$ produce the instances of 1patterns having $f_{r}$ as reference feature. Consider a featureset $\left\langle F_{i}, t_{i}\right\rangle \in \mathcal{S}_{i}^{f}$. For each feature $f_{j} \in F_{i}$ an element $\left(o_{i}, t_{i}\right)$ is added to list IT List $_{f_{r}}\left(f_{j}\right)$, indicating that there is an object of feature $f_{j}$ close to object $o_{i}$ of feature $f_{r}$ at time window $\left[t_{i}, t_{i}+w\right)$. By sliding a window $W$ over IT List $_{f_{r}}\left(f_{j}\right)$, we can compute the supports of the 1-pattern $\left(f_{j}\right)$ referencing $f_{r}$. The ITlists are then used to find the frequent 1-patterns $L_{1}$ (Line 2 of the algorithm).

For counting the instances of a longer candidate pattern $P$ (procedure MJ_count_cand), we slide a $W$-window along the two ITLists of the two subpatterns $P_{1}$ and $P_{2}$ that generate $P$, and merge-join the lists to create $\operatorname{ITList}_{f_{r}}(P)$. For every position $t$ of $W$, such that $\operatorname{ITList}_{f_{r}}\left(P_{1}\right)$ and IT List $_{f_{r}}\left(P_{2}\right)$ contain entries of the same $o_{i}$ and these en- 
tries qualify the pattern constraints, a new instance is generated for ITList $f_{r}(P)$. Entries in the ITList of a long pattern with $k$ units is a list of $\left(o_{i}, I_{g_{1}} \cdot t_{s}, \ldots, I_{g_{k}} \cdot t_{s}\right)$. We distinguish three cases for this merge-join process:

- $P_{1}$ and $P_{2}$ contain collocation units that are exactly the same in $P$. For example, $P_{1}=\left(f_{1}\right) \rightarrow\left(f_{2}\right)$, $P_{2}=\left(f_{1}\right) \rightarrow\left(f_{3}\right), P=\left(f_{1}\right) \rightarrow\left(f_{2}\right) \rightarrow\left(f_{3}\right)$. In this case, IT List $f_{r}\left(P_{1}\right)$, IT List $f_{r}\left(P_{2}\right)$ are joined according to the $t_{s}$ time of the common unit, while the rest of the temporal constraints are verified.

- $P_{1}$ and $P_{2}$ contain collocation units that are joined in $P$. E.g, $P_{1}=\left(f_{1}, f_{2}\right), P_{2}=\left(f_{2}\right) \rightarrow\left(f_{3}\right), P=\left(f_{1}, f_{2}\right) \rightarrow$ $\left(f_{3}\right)$. In this case, IT List $f_{r}\left(P_{1}\right)$, IT List $_{f_{r}}\left(P_{2}\right)$ are joined according to the $t_{s}$ time of the joined units, while the rest of the temporal constraints are verified.

- $P_{1}$ and $P_{2}$ do not have common or joined units. For example, $P_{1}=\left(f_{1}\right), P_{2}=\left(f_{2}\right), P=\left(f_{1}\right) \rightarrow$ $\left(f_{2}\right)$. In this case, we perform a band-join [2] between IT List $f_{r}\left(P_{1}\right)$ and IT List $f_{r}\left(P_{2}\right)$ to produce IT List $_{f_{r}}(P)$. The band-join is a straightforward extension of the merge join algorithm that replaces the equality condition by a maximum difference constraint (maximum time difference $W$ in our example).

\section{Experimental Evaluation}

This section experimentally evaluates the performance of the proposed algorithms based on synthetically generated data due to the lack of real data. All experiments were run on a Pentium III Xeon $700 \mathrm{MHz}$ workstation with $4096 \mathrm{MB}$ RAM, running Solaris 9x86. The generator takes as input the following parameters: $|\mathcal{F}|$, the number of features; $\ell$, the maximal length of the generated episodes; $n$, the number of sequences (i.e., objects); $m$, the maximal length of every sequence; $\epsilon, w, W$, and min_sup, which have the same meaning as that in the problem definition. Given these parameters, we generate $n$ trajectories, each of which is assigned to a type in $\mathcal{F}$ while making sure that the generated trajectories instantiate collocation episodes. The default values of the data generation parameters are $n=500, m=2000, w=2$, $W=20,|\mathcal{F}|=40, \ell=7, \epsilon=1 \%$ and min_sup $=0.03$. Unless otherwise stated, we use the same parameter values in data generation and data mining.

Performance evaluation Our methods discover the collocation episodes in two steps; first, close feature sets are found and then longer patterns are extracted from them. For the first step, apart from the proposed hash-based method, we implement a naive one by linearly scanning all other trajectories. For the second step, besides implementing the two algorithms Apriori and MJ, we also developed a non-optimized version of the Apriori algorithm, which does not employ the optimized counting approach shown in Figure 7. We compare the performance of four methods. Apriori-base applies linear scan in the first step and non-optimized Apriori for finding the patterns. Apriori-noprune, Apriori, and $M J$ use the hash-based method in the first step, and non-optimized Apriori, optimized Apriori, and MJ respectively, in the second step. The difference between the linear scan method and the hash-based approach in the first step can be seen by comparing Apriori-base and Apriori-noprune. The difference between finding collocations using the transformed sequences and the ITLists could be observed from Apriori and MJ. Fi- nally, by comparing Apriori-noprune with Apriori we can see the effect of optimized support counting in Apriori.

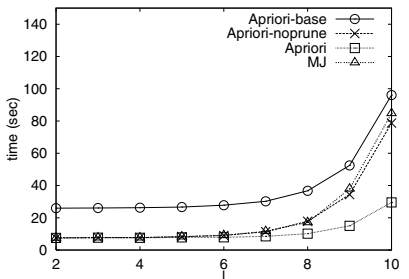

Figure 8: Time vs. $\ell$

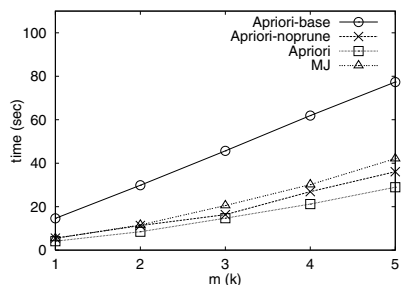

Figure 9: Time vs. $m$
Figure 8 shows that the mining cost increases with the maximal length $\ell$ of the generated episodes. In addition, since the number of candidates in each level grows exponentially to $\ell$, the cost varies slightly for smaller $\ell$, and increases sharply when $\ell$ becomes large. However, the optimized counting of Apriori slows down this exponential growth. Figure 9 illustrates the scalability of the methods over the maximal length $m$ of the sequences. It shows that the mining cost grows nearly linear to $m$, exhibiting good scalability over the data volume. For changing $n$, the linear changing trend could be observed. To summarize, for finding close feature pairs, the hash-based technique is much faster than the linear scan method, whereas for discovering collocation episodes from feature sets, the Apriori method with the counting optimization technique performs best. On the other hand, in most cases, MJ is not as efficient as Apriori, due to the large volume of generated and joined ITLists.

\section{Conclusion}

In this paper, we studied the problem of discovering frequent collocation episodes from spatiotemporal data. We provided a novel and carefully designed definition of this new and important mining problem. In addition, we designed an efficient two-phase mining methodology. In the first phase, a hash-based technique is used to convert the original trajectories to sequences of close features to the corresponding object. In the second phase, an Apriori-based technique is devised to discover the frequent episodes. We showed by experimentation that the best combination of techniques in both phases is efficient and scalable.

\section{References}

[1] G. Andrienko, D. Malerba, M. May, and M. Teisseire, editors. ECML/PKDD Workshop on Mining Spatio-Temporal Data, 2005.

[2] D. J. DeWitt, J. F. Naughton, and D. A. Schneider. An evaluation of non-equijoin algorithms. In $V L D B, 1991$

[3] M. Hadjieleftheriou, G. Kollios, P. Bakalov, and V. J. Tsotras. Complex spatio-temporal pattern queries. In $V L D B, 2005$.

[4] J. Lin, E. J. Keogh, A. W.-C. Fu, and H. V. Herle. Approximations to magic: Finding unusual medical time series. In 18th IEEE Symp. on Computer-Based Medical Systems (CBMS), 2005.

[5] H. Mannila, H. Toivonen, and A. I. Verkamo. Discovery of frequent episodes in event sequences. Data Min. Knowl. Discov., 1(3):9, 1997.

[6] S. Shekhar and Y. Huang. Discovering spatial co-location patterns: A summary of results. In SSTD, 2001

[7] R. Srikant and R. Agrawal. Mining sequential patterns: Generalizations and performance improvements. In EDBT, 1996.

[8] J. Wang, W. Hsu, and M.-L. Lee. A framework for mining topological patterns in spatio-temporal databases. In CIKM, 2005.

[9] H. Yang, S. Parthasarathy, and S. Mehta. A generalized framework for mining spatio-temporal patterns in scientific data. In KDD, 2005. 